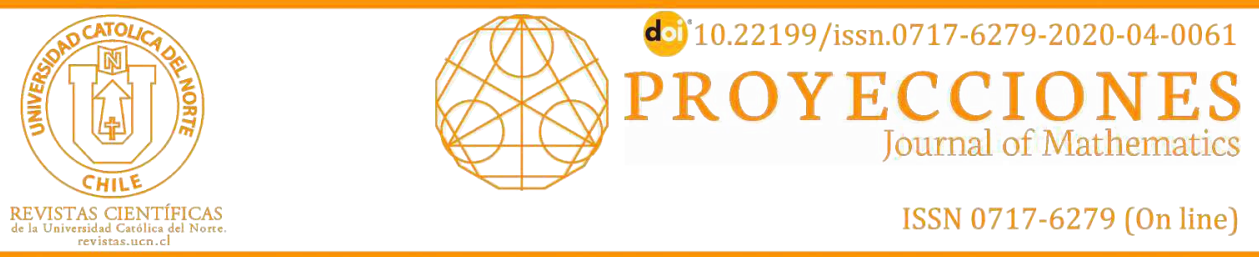

\title{
Topological properties of four types of porphyrin dendrimers
}

\author{
Abdul Jalil M. Khalaf ${ }^{1}$ (D) orcid.org/0000-0002-2447-6666 \\ Aisha Javed $^{2}$ (D orcid.org/0000-0001-5465-8922 \\ Muhammad Kamran Jamil ${ }^{3}$ (0) orcid.org/0000-0001-5715-9299 \\ Mehdi Alaeiyan ${ }^{4}$ (1) orcid.org/0000-0003-2185-5967 \\ Mohammad Reza Farahani ${ }^{5}$ (1) orcid.org/0000-0003-2969-4280 \\ ${ }^{1}$ University of Kufa, Dept. of Mathematics, Najaf, Iraq. \\ -abduljaleel.khalaf@uokufa.edu.iq \\ ${ }^{2}$ Government College University, Abdus Salam School of Mathematical Sciences, Lahore, Pakistan. \\ -aaishajaved@gmail.com \\ ${ }^{3}$ Riphah International University, RICAS, Dept. of Mathematics, Lahore, Pakistan. \\ m.kamran.sms@gmail.com \\ Iran University of Science and Technology, Dept.of Mathematics, Tehran, Iran. \\ 4@alaeiyan@iust.ac.ir ; 5 mrfarahani88@gmail.com
}

Received: February 2020 | Accepted: June 2020

\section{Abstract:}

A chemical compound can be represented as a chemical graph. A topological index of a (chemical) graph is a numeric value of a graph which characterize its topology and is usually graph invariant. The Zagreb indices, Randić index and sum-connectivity indices are useful in the study of antiinflammatory activities, boiling point, molecular complexity heterosystems of certain chemical instances, and in elsewhere. In this paper, we calculate the mentioned topological indices of some infinite classes of prophyrin dendrimers.

Keywords: Dendrimers; Zagreb indices; Randić index; Sum-connectivity index.

MSC (2020): 05C10, 05C12, 05C15, 05C22, 05C31.

\section{Cite this article as (IEEE citation style):}

A. J. M. Khalaf, A. Javed, M. K. Jamil, M. Alaeiyan, and M. Reza Farahani, "Topological properties of four types of porphyrin dendrimers", Proyecciones (Antofagasta, On line), vol. 39, no. 4, pp. 979-993, Aug. 2020, doi: 10.22199/issn.0717-6279-2020-040061.

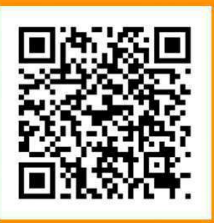

Article copyright: (c) 2020 Abdul Jalil M. Khalaf, Aisha Javed, Muhammad Kamran Jamil, Mehdi Alaeiyan and Mohammad Reza Farahani. This is an open access article distributed under the terms of the Creative Commons License, which permits unrestricted use and distribution provided the original author and source are credited. 


\section{Introduction}

The properties of a molecule are tightly connected to its characteristics is one of the fundamental concepts in chemistry. In this connection, graph theory has been successfully applied $[5,11,23]$.

Chemical graph theory is a branch of mathematical chemistry. It is concerned with handling chemical graphs that represent chemical systems. Hence, chemical graph theory deals with analyses of all consequences of connectivity in a chemical system. In other words, chemical graph theory is concerned with all aspects of the application of graph theory to chemistry area.

Chemists employ various types of designations and formulas when they want to communicate information about chemical compounds and their structures. In spite of this fact, most of the names and formulas have to direct, immediate or explicit mathematical meaning. It has been found to be a useful tool in QSAR (Quantitative Structure Activity Relationship) and QSPR (Quantitative Structure Property Relationship). A lot of studies have been done relating to the above mentioned fields by using what are called topological indices [7, 16, 24, 25].

A chemical structure can be represented by using graph theory, where vertices denote atoms and edges denotes molecular bonds. A topological index is a numeric number that indicates some useful information about the molecular structure. It is the numerical invariants of a molecular graph and is useful to correlate with their bioactivity and physiochemical properties. Researchers have found the topological index to be a powerful and useful tool in the description of molecular structure. Some applications related to topological indices of molecular graphs are included in the reference list $[10,15,22,26]$.

Dendrimers are among the most complex chemical and interesting structures and hyper-branched macromolecules, with a precise tailored architecture. Dendrimers have gained a wide range of application in supramolecular chemistry, particularly in host guest reactions and the self-assembly process [21].

The introduction of porphyrins into dendritic structures began in the early days of dendrimer chemistry, mainly as core entities, due to the morphological resemblance of these macrostructures to natural hemoproteins. Over 
the years, porphyrin macrocycles have also been introduced as dendritic surface groups or within the dendritic branches [20].

Let $G(V(G), E(G))$ be a graph where $V(G)$ is the vertex set and $E(G)$ is the edge set of the graph $G$. The degree, $\operatorname{deg}(v)$, of the vertex $v$ in a graph $G$ is the number of edges of $G$ incident with $v$ in $G$. The length of the shortest path in a graph $G$ between vertices $u$ and $v$ is the distance, $d(u, v)$, between $u$ and $v$.

A graph can be represented by a polynomial, numerical value, or a matrix form. There are certain types of topological indices, which are mainly eccentric based, degree based and distance based. In this article, we deal with degree-based topological indices.

Zagreb index $\left(M_{1}\right)$ is one of the most oldest and important degree based topological indices. In 1972, Gutman and Trinajstic̀ proposed this topological index [9]. After that, they introduced the second Zagreb index $\left(M_{2}\right)$ [8]. These indices are defined as follows

$$
\begin{gathered}
M_{1}(G)=\sum_{v \in V(G)} d^{2}(G)=\sum_{u v \in E(G)}(d(u)+d(v)) \\
M_{2}(G) \\
=\sum_{u v \in E(G)} d(u) d(v)
\end{gathered}
$$

In 1975, M. Randic̀ introduced the Randic̀ index $(R)$. This topological index has great importance in QSPR/QSAR. The Randic index of a graph $G$ is defined as

$$
R(G)=\sum_{u v \in E(G)} \frac{1}{\sqrt{d(u) d(v)}}
$$

A closely related topological index named as sum-connectivity index was proposed by Zhou et. al. [27]. The sum-connectivity index of a graph $G$ is obtained from Randic̀ index by replacing the term $d(u) d(v)$ by $d(u)+d(v)$. In QSPR/QSAR, the sum connectivity index shows some better results in some aspects. It is defined as

$$
\chi(G)=\sum_{u v \in E(G)} \frac{1}{\sqrt{d(u)+d(v)}}
$$

The molecular graph of dendrimers structures is denoted by $G_{n}$, where $n$ is the growth stages and $n \geq 0$.

For detailed study computation of topological indices of molecular structures we refer $[1,2,3,4,12,13,6,18,19]$. 


\section{Four Layered Prophyrin Core Dendrimers}

The majority of porphyrin dendrimers reported to date possess a (metallo) porphyrin unit as the interior core moiety only. Pooneering work in the field of porphyrin dendrimers was performed in laboratories of Aida, Diederich and Suslick. The first example was described by Aida and co-workers in 1993 [14]. Figure 2.1 shows the Aida's four layered porphyrin core dendrimer. In this section, we compute the certain topological indices of the four layered porphyrin core dendrimers.

The molecular graph shown in Figure 2.1 contain $136 \cdot 2^{n}-15$ vertices (aotms) and $152 \cdot 2^{n}-12$ edges (bonds). There are four types of vertices based on the degree, vertices with degree 1,2, 3 and 4 . We partition the edge set of the molecular graph of Aida's four layered prophyrin core dendrimer based on the degree of end vertices of each edge. Table 2.1 illustrate this partition.

\begin{tabular}{|c|c|c|c|c|c|}
\hline \hline$(d(u), d(v)), n \geq 1$ & $(2,2)$ & $(2,3)$ & $(1,3)$ & $(3,3)$ & $(3,4)$ \\
\hline number of edges & $32 \cdot 2^{n}-4$ & $112 \cdot 2^{n}-32$ & $8 \cdot 2^{n}$ & 20 & 4 \\
\hline \hline
\end{tabular}

Table 2.1: Partition of the edge set of Aida's four layered prophyrin core dendrimer based on the degree of end vertices of each edge.

Let $G_{n}$ be the molecular graph of the four layered prophyrin core dendrimer, then the Zagreb indices of $G_{n}$ is equal to

$$
\begin{aligned}
& M_{1}\left(G_{n}\right)=45 \cdot 2^{(n+4)}-28 \\
& M_{2}\left(G_{n}\right)=103 \cdot 2^{(n+3)}+20
\end{aligned}
$$

Proof. The graph $G_{n}$ contains the vertices of degrees $1,2,3$ and 4 . We found the edge partition of the form $(2,2),(2,3),(1,3),(3,3)$ and $(3,4)$ for $G_{n}$ based on the degree of end vertices of each edge. Table 2.1 explains such partition. By using the Table 2.1, we can compute the first and second Zagreb indices of $G_{n}$, as follows

$$
\begin{aligned}
M_{\left(G_{n}\right)} & =\sum_{u v \in E\left(G_{n}\right)}(d(u)+d(v)) \\
& =\left(32 \cdot 2^{n}-4\right)(2+2)+\left(112 \cdot 2^{n}-32\right)(2+3)+\left(8 \cdot 2^{n}\right)(1+3)+20(3+3)+4(3+4) \\
& =45 \cdot 2^{(n+4)}-28
\end{aligned}
$$




$$
\begin{aligned}
M_{\left(G_{n}\right)} & =\sum_{u v \in E\left(G_{n}\right)} d(u) d(v) \\
& =\left(32 \cdot 2^{n}-4\right)(2 \cdot 2)+\left(112 \cdot 2^{n}-32\right)(2 \cdot 3)+\left(8 \cdot 2^{n}\right)(1 \cdot 3)+20(3 \cdot 3)+4(3 \cdot 4) \\
& =103 \cdot 2^{(n+3)}+20
\end{aligned}
$$

Let $G_{n}$ be the molecular graph of the four layered prophyrin core dendrimer, then the Randic index of $G_{n}$ is given as

$$
R\left(G_{n}\right)=\frac{2}{3}\left((6+3 \sqrt{3}+7 \sqrt{6}) 2^{(n+2)}+7+\sqrt{3}-8 \sqrt{6}\right)
$$

Proof. The edge partition of the graph $G_{n}$ based on the degree of end vertices of each edge is shown in Table 2.1. Now we apply the formula of Randic index to obtain the result

$$
\begin{aligned}
R\left(G_{n}\right) & =\sum_{u v \in E\left(G_{n}\right)} \frac{1}{\sqrt{d(u) d(v)}} \\
& =\left(32 \cdot 2^{n}-4\right) \frac{1}{\sqrt{2 \cdot 2}}+\left(112 \cdot 2^{n}-32\right) \frac{1}{\sqrt{2 \cdot 3}}+\left(8 \cdot 2^{n}\right) \sqrt{1 \cdot 3}+20 \frac{1}{\sqrt{3 \cdot 3}}+4 \frac{1}{\sqrt{3 \cdot 4}} \\
& =\frac{2}{3}\left((6+3 \sqrt{3}+7 \sqrt{6}) 2^{(n+2)}+7+\sqrt{3}-8 \sqrt{6}\right)
\end{aligned}
$$

Let $G_{n}$ be the molecular graph of the four layered prophyrin core dendrimer, then the sum-connectivity index of $G_{n}$ is

$$
\chi\left(G_{n}\right)=(2+7 \sqrt{5}) 2^{(n+4)}-2+10 \sqrt{\frac{2}{3}}-\frac{32}{\sqrt{5}}+\frac{4}{\sqrt{7}}
$$

Proof. By using the definition of sum-connectivity index and the Table 2.1 we can obtain the required result. Since

$$
\chi\left(G_{n}\right)=\sum_{u v \in E\left(G_{n}\right)} \frac{1}{\sqrt{d(u)+d(v)}}
$$

this implies that

$$
\begin{aligned}
\chi\left(G_{n}\right) & =\left(32 \cdot 2^{n}-4\right) \frac{1}{\sqrt{2+2}}+\left(112 \cdot 2^{n}-32\right) \frac{1}{\sqrt{2+3}}+\left(8 \cdot 2^{n}\right) \sqrt{1} \sqrt{1+3}+ \\
& 20 \frac{1}{\sqrt{3+3}}+4 \frac{1}{\sqrt{3+4}} \\
& =(2+7 \sqrt{5}) 2^{(n+4)}-2+10 \sqrt{\frac{2}{3}}-\frac{32}{\sqrt{5}}+\frac{4}{\sqrt{7}}
\end{aligned}
$$




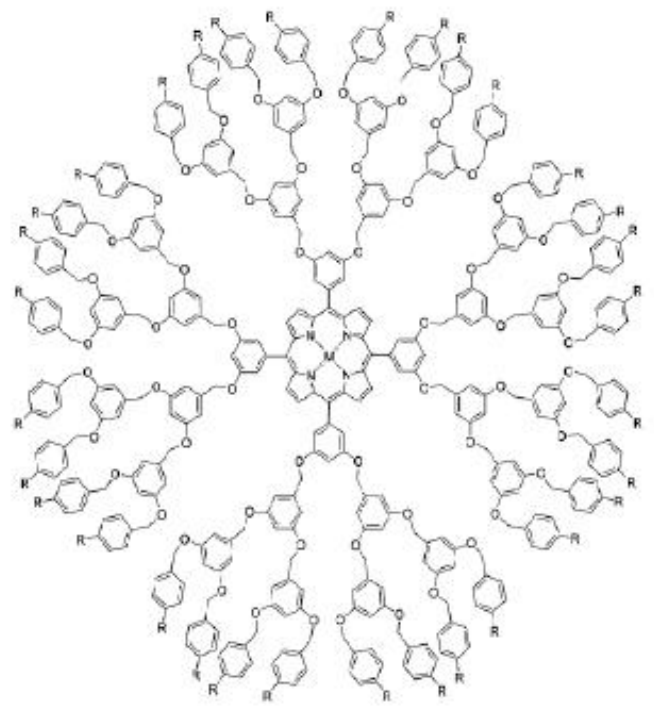

Figure 2.1: Molecular graph of four Layered Prophyrin Core Dendrimer.

\section{Porphyrin-Core Denrimer With $n$ Layers of Internal Tria- zole Units}

Very recently, several dendritic porphyrins in which benzyl ether dendritic azides were clicked to an acetylene terminated Znporphyrin core through $\mathrm{Cu}$-catalyzed cycloaddition reactions (52-90 to afford 1,2,3-triazole links were designed by Kimura et. al. [17]. A molecular graph of porphyrin-core denrimer with $n$ layers of internal triazole units is shown in Fig. 3.1.

From Figure 3.1 one can notice that the graph have $256 \cdot 2^{n}-63$ vertices (aotms) and $288 \cdot 2^{n}-68$ edges (bonds). There are vertices with degree 1, 2, 3 and 4 . We partition the edge set of the molecular graph of porphyrin-core dendrimer with $n$ layers of internal triazole units based on the degree of end vertices of each edge. Table 3.1 illustrate this partition.

\begin{tabular}{|c|c|c|c|c|c|}
\hline \hline$(d(u), d(v)), n \geq 1$ & $(2,2)$ & $(2,3)$ & $(1,3)$ & $(3,3)$ & $(3,4)$ \\
\hline number of edges & $48 \cdot 2^{n}-12$ & $208 \cdot 2^{n}-80$ & $24 \cdot 2^{n}$ & $8 \cdot 2^{n}+20$ & 4 \\
\hline \hline
\end{tabular}

Table 3.1: Partition of the edge set of porphyrin-core dendrimer with $N$ layers of internal triazole units based on the degree of end vertices of each edge. 


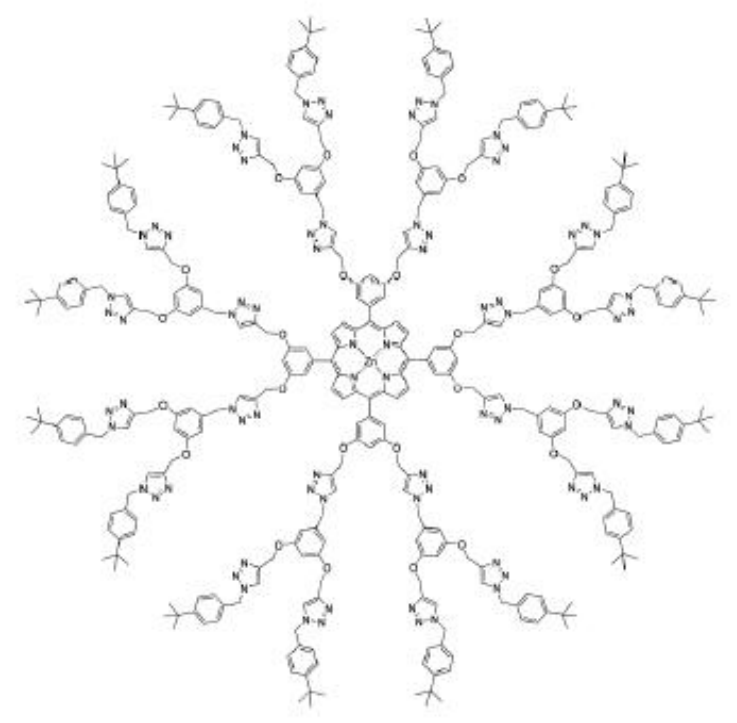

Figure 3.1: Porphyrin-core dendrimer with $n=2$ layers of internal triazole units.

Let $H_{n}$ be the molecular graph of the porphyrin-core denrimer with $n$ layers of internal triazole units, then the Zagreb indices of $H_{n}$ is equal to

$$
\begin{aligned}
& M_{1}\left(H_{n}\right)=43 \cdot 2^{(n+5)}-300 \\
& M_{2}\left(H_{n}\right)=99 \cdot 2^{(n+4)}-300
\end{aligned}
$$

Proof. The graph $H_{n}$ contains the vertices of degrees 1,2,3 and 4. We found the edge partition of the form $(2,2),(2,3),(1,3),(3,3)$ and $(3,4)$ for $H_{n}$ based on the degree of end vertices of each edge. Table 3.1 explains such partition. By using the Table 3.1, we can compute the first and second Zagreb indices of $H_{n}$, as follows

$$
\begin{aligned}
M_{1}\left(H_{n}\right) & =\sum_{u v \in E\left(H_{n}\right)}(d(u)+d(v)) \\
& =\left(48 \cdot 2^{n}-12\right)(2+2)+\left(208 \cdot 2^{n}-80\right)(2+3)+\left(24 \cdot 2^{n}\right)(1+3)+ \\
& \left(8 \cdot 2^{n}+20\right)(3+3)+4(3+4) \\
& =43 \cdot 2^{(n+5)}-300 \\
& =\sum_{u v \in E\left(H_{n}\right)} d(u) d(v) \\
& =\left(48 \cdot 2^{n}-12\right)(2 \cdot 2)+\left(208 \cdot 2^{n}-80\right)(2 \cdot 3)+\left(24 \cdot 2^{n}\right)(1 \cdot 3)+ \\
& \left(8 \cdot 2^{n}+20\right)(3 \cdot 3)+4(3 \cdot 4) \\
& =99 \cdot 2^{(n+4)}-300
\end{aligned}
$$


Let $H_{n}$ be the molecular graph of the porphyrin-core denrimer with $n$ Layers of internal triazole units, then the Randic index of $H_{n}$ is given as

$$
R\left(H_{n}\right)=\frac{2}{3}\left((10+3 \sqrt{3}+13 \sqrt{6}) 2^{(n+2)}+1+\sqrt{3}-20 \sqrt{6}\right)
$$

Proof. The edge partition of the graph $H_{n}$ based on the degree of end vertices of each edge is shown in Table 3.1. Now we apply the formula of Randic index to obtain the result

$$
\begin{aligned}
R\left(H_{n}\right) & =\sum_{u v \in E\left(G_{n}\right)} \frac{1}{\sqrt{d(u) d(v)}} \\
& =\left(48 \cdot 2^{n}-12\right) \frac{1}{\sqrt{2 \cdot 2}}+\left(208 \cdot 2^{n}-80\right) \frac{1}{\sqrt{2 \cdot 3}}+\left(24 \cdot 2^{n}\right) \frac{1}{\sqrt{1 \cdot 3}}+ \\
& \left(8 \cdot 2^{n}+20\right) \frac{1}{\sqrt{3 \cdot 3}}+4 \frac{1}{\sqrt{3 \cdot 4}} \\
& =\frac{2}{3}\left((10+3 \sqrt{3}+13 \sqrt{6}) 2^{(n+2)}+1+\sqrt{3}-20 \sqrt{6}\right)
\end{aligned}
$$

Let $H_{n}$ be the molecular graph of the porphyrin-core denrimer with $n$ Layers of internal triazole units, then the sum-connectivity index of $H_{n}$ is

$$
\chi\left(H_{n}\right)=9 \cdot 2^{(n+2)}-6+\frac{16\left(13 \cdot 2^{n}-5\right)}{\sqrt{5}}+\frac{2^{(n+3)}+20}{\sqrt{6}}+\frac{4}{\sqrt{7}}
$$

Proof. By using the definition of sum-connectivity index and the Table 2.1 we can obtain the required result. Since

$$
\chi\left(H_{n}\right)=\sum_{u v \in E\left(H_{n}\right)} \frac{1}{\sqrt{d(u)+d(v)}}
$$

this implies that

$$
\begin{aligned}
\chi\left(H_{n}\right) & =\left(48 \cdot 2^{n}-12\right) \frac{1}{\sqrt{2+2}}+\left(208 \cdot 2^{n}-80\right) \frac{1}{\sqrt{2+3}}+\left(24 \cdot 2^{n}\right) \frac{1}{\sqrt{1+3}}+ \\
& \left(8 \cdot 2^{n}+20\right) \frac{1}{\sqrt{3+3}}+4 \frac{1}{\sqrt{3+4}} \\
& =9 \cdot 2^{(n+2)}-6+\frac{16\left(13 \cdot 2^{n}-5\right)}{\sqrt{5}}+\frac{2^{(n+3)}+20}{\sqrt{6}}+\frac{4}{\sqrt{7}}
\end{aligned}
$$

\section{Proteo-dendrimers}

In Proteo-dendrimers three hydrophobic Frchet dendrons with a hydrophilic polyether surface are combined with a poly(glutamic acid) dendron around a fluorescent Znporphyrin core. The molecular graph of Proteo-dendrimer is shown in Fig. 4.1. 
The molecular graph of proteo-dendrimers have $154 \cdot 2^{n}-62$ vertices (aotms) and $168 \cdot 2^{n}$ edges (bonds). There are vertices with degree 1, 2, 3 and 4. The partitioning of the edge set of the molecular graph of proteodendrimers based on the degree of end vertices of each edge. Table 4.1 illustrate this partition.

\begin{tabular}{|c|c|c|c|c|c|c|}
\hline \hline$(d(u), d(v)), n \geq 1$ & $(2,1)$ & $(2,2)$ & $(2,3)$ & $(1,3)$ & $(3,3)$ & $(3,4)$ \\
\hline number of edges & $6 \cdot 2^{n}$ & $56 \cdot 2^{n}-1$ & $98 \cdot 2^{n}-22$ & $6 \cdot 2^{n}-1$ & $2 \cdot 2^{n}+20$ & 4 \\
\hline \hline
\end{tabular}

Table 4.1: Partition of the edge set of proteo-dendrimers based on the degree of end vertices of each edge.

Let $I_{n}$ be the molecular graph of the Proteo-dendrimers, then the Zagreb indices of $I_{n}$ is equal to

$$
\begin{gathered}
M_{1}\left(I_{n}\right)=6\left(2^{(n+7)}+5\right) \\
M_{2}\left(I_{n}\right)=215 \cdot 2^{(n+2)}+89
\end{gathered}
$$

Proof. The molecular graph of proteo-dendirmer contains the $154 \cdot 2^{n}-$ 62 vertices of degrees one, two, three and four. Based on the end degrees of each edge the partition is shown in Table 4.1. Since

$$
\begin{aligned}
M_{1}\left(I_{n}\right) & =\sum_{u v \in E\left(I_{n}\right)}(d(u)+d(v)) \\
& =\left(6 \cdot 2^{n}\right)(2+1)+\left(56 \cdot 2^{n}-1\right)(2+2)+\left(98 \cdot 2^{n}-22\right)(2+3)+\left(6 \cdot 2^{n}-1\right)(1+3) \\
& +\left(2 \cdot 2^{n}+20\right)(3+3)+4(3+4) \\
& =6\left(2^{(n+7)}+5\right) \\
M_{2}\left(I_{n}\right) & =\sum_{u v \in E\left(H_{n}\right)} d(u) d(v) \\
& =\left(6 \cdot 2^{n}\right)(2 \cdot 1)+\left(56 \cdot 2^{n}-1\right)(2 \cdot 2)+\left(98 \cdot 2^{n}-22\right)(2 \cdot 3)+\left(6 \cdot 2^{n}-1\right)(1 \cdot 3)+ \\
& \left(2 \cdot 2^{n}+20\right)(3 \cdot 3)+4(3 \cdot 4) \\
& =215 \cdot 2^{(n+2)}+89
\end{aligned}
$$

Let $I_{n}$ be the molecular graph of the Proteo-dendrimers, then the Randic index of $I_{n}$ is given as

$$
R\left(I_{n}\right)=\frac{\left(6 \cdot 2^{n}\right)}{\sqrt{2}}+\frac{\left(172 \cdot 2^{n}+37\right)}{6}+\frac{\left(98 \cdot 2^{n}-22\right)}{\sqrt{6}}+\frac{\left(6 \cdot 2^{n}-1\right)}{\sqrt{3}}+\frac{4}{\sqrt{12}}
$$


Proof. By definition of Randic̀ index and the Table 4.1, we have

$$
\begin{aligned}
R\left(I_{n}\right) & =\sum_{u v \in E\left(H_{n}\right)} \frac{1}{\sqrt{d(u) d(v)}} \\
& =\frac{\left(6 \cdot 2^{n}\right)}{\sqrt{2 \cdot 1}}+\frac{\left(56 \cdot 2^{n}-1\right)}{\sqrt{2 \cdot 2}}+\frac{\left(98 \cdot 2^{n}-22\right)}{\sqrt{2 \cdot 3}}+\frac{\left(6 \cdot 2^{n}-1\right)}{\sqrt{1 \cdot 3}}+\frac{\left(2 \cdot 2^{n}+20\right)}{\sqrt{3 \cdot 3}}+\frac{4}{\sqrt{3 \cdot 4}} \\
& =\frac{\left(6 \cdot 2^{n}\right)}{\sqrt{2}}+\frac{\left(172 \cdot 2^{n}+37\right)}{6}+\frac{\left(98 \cdot 2^{n}-22\right)}{\sqrt{6}}+\frac{\left(6 \cdot 2^{n}-1\right)}{\sqrt{3}}+\frac{4}{\sqrt{12}}
\end{aligned}
$$

Let $I_{n}$ be the molecular graph of the Proteo-dendrimers, then the sumconnectivity index of $I_{n}$ is

$$
\chi\left(I_{n}\right)=31 \cdot 2^{n}-2+\frac{\left(6 \cdot 2^{n}\right)}{\sqrt{3}}+\frac{98 \cdot 2^{n}-22}{\sqrt{5}}+\frac{\left(2 \cdot 2^{n}+20\right)}{\sqrt{6}}+\frac{4}{\sqrt{7}}
$$

Proof. Result can be obtained by applying Table 4.1 in definition of sum-connectivity index as

$$
\begin{aligned}
\chi\left(I_{n}\right) & =\sum_{u v \in E(I n)} \frac{1}{\sqrt{d(u)+d(v)}} \\
& =\frac{\left(6 \cdot 2^{n}\right)}{\sqrt{2+1}}+\frac{\left(56 \cdot 2^{n}-1\right)}{\sqrt{2+2}}+\frac{98 \cdot 2^{n}-22}{\sqrt{2+3}}+\frac{\left(6 \cdot 2^{n}-1\right)}{\sqrt{1+3}}+\frac{\left(2 \cdot 2^{n}+20\right)}{\sqrt{3+3}}+\frac{4}{\sqrt{3+4}} \\
& =31 \cdot 2^{n}-2+\frac{\left(6 \cdot 2^{n}\right)}{\sqrt{3}}+\frac{98 \cdot 2^{n}-22}{\sqrt{5}}+\frac{\left(2 \cdot 2^{n}+20\right)}{\sqrt{6}}+\frac{4}{\sqrt{7}}
\end{aligned}
$$

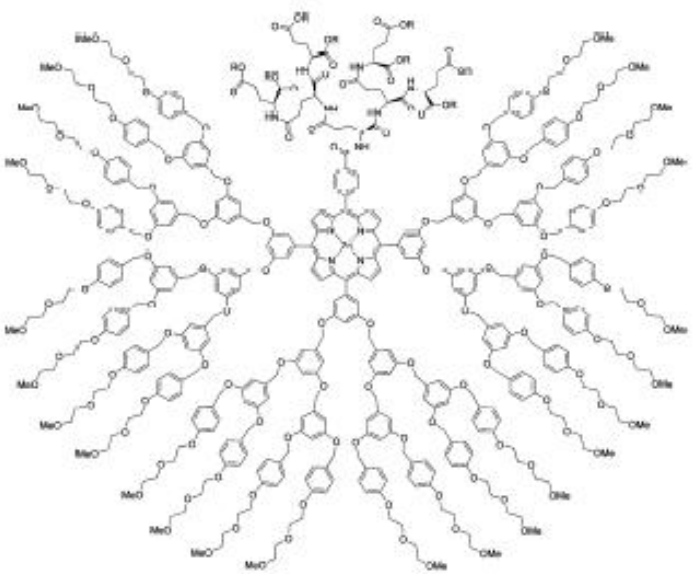

Figure 4.1: Molecular graph of Proteo-dendrimers. 


\section{Dendritic Iron (II) Porphyrins}

The molecular graph of dendtitic iron (II) porphyrins is shown in Fig. 5.1. This molecular structure has $168 \cdot 2^{n}-4$ vertices (atoms) and $184 \cdot 2^{n}-6$ edges (bonds). The vertices have degrees 1,2,3 and 4. Based on the degrees of the end vertices of each edge we partitioned the edge set. This partitioning is shown in Table 5.1.

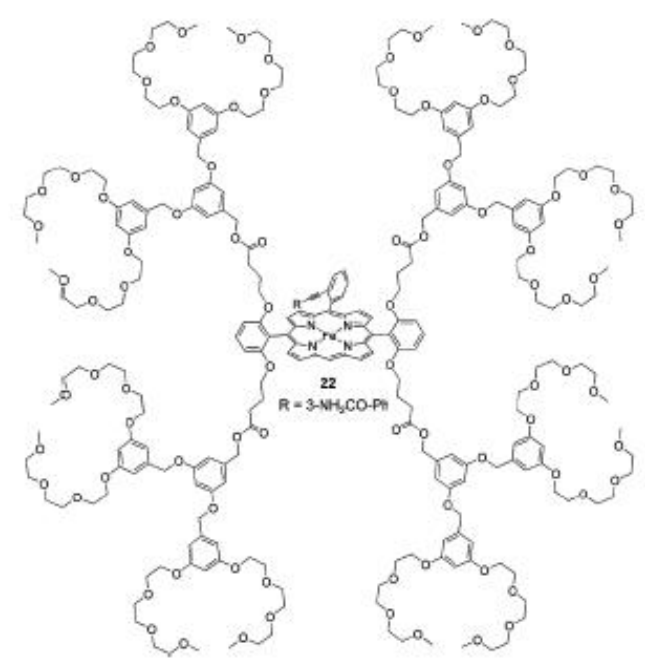

Figure 5.1: First generation of dendritic iron (II) porphyrin.

\begin{tabular}{|c|c|c|c|c|c|c|}
\hline \hline$(d(u), d(v)), n \geq 1$ & $(1,2)$ & $(2,2)$ & $(2,3)$ & $(1,3)$ & $(3,3)$ & $(3,4)$ \\
\hline number of edges & $8 \cdot 2^{n}$ & $92 \cdot 2^{n}-5$ & $80 \cdot 2^{n}-28$ & $4 \cdot 2^{n}-3$ & 22 & 8 \\
\hline \hline
\end{tabular}

Table 5.1: Partition of the edge set of dendritic iron (II) porphyrin based on the degree of end vertices of each edge.

Let $J_{n}$ be the molecular graph of the Proteo-dendrimers, then the Zagreb indices of $J_{n}$ is

$$
\begin{aligned}
& M_{1}\left(J_{n}\right)=8\left(101 \cdot 2^{n}+2\right) \\
& M_{2}\left(J_{n}\right)=219 \cdot 2^{(n+2)}+97
\end{aligned}
$$

Proof. The molecular graph of dendritic iron (II) porphyrin consist of $168 \cdot 2^{n}-4$ vertices. Based on the end degrees of each edge the partition of edge set is illustrated in Table 5.1. Since 


$$
\begin{aligned}
M_{1}\left(J_{n}\right) & =\sum_{u v \in E\left(J_{n}\right)}(d(u)+d(v)) \\
& =\left(8 \cdot 2^{n}\right)(1+2)+\left(92 \cdot 2^{n}-5\right)(2+2)+\left(80 \cdot 2^{n}-28\right)(2+3)+\left(4 \cdot 2^{n}-3\right)(1+3) \\
& +(22)(3+3)+8(3+4) \\
& =8\left(101 \cdot 2^{n}+2\right) \\
M_{2}\left(J_{n}\right) & =\sum_{u v \in E\left(J_{n}\right)} d(u) d(v) \\
& =\left(8 \cdot 2^{n}\right)(2 \cdot 1)+\left(92 \cdot 2^{n}-5\right)(2 \cdot 2)+\left(80 \cdot 2^{n}-28\right)(2 \cdot 3)+\left(4 \cdot 2^{n}-3\right)(1 \cdot 3)+ \\
& (22)(3 \cdot 3)+8(3 \cdot 4) \\
& =219 \cdot 2^{(n+2)}+97
\end{aligned}
$$

Let $J_{n}$ be the molecular graph of the dendritic iron (II) porphyrin, then the Randic index of $I_{n}$ is equal to

$$
R\left(J_{n}\right)=\frac{2^{n+3}}{\sqrt{2}}+\frac{\left(276 \cdot 2^{n}+29\right)}{6}+\frac{\left(80 \cdot 2^{n}-28\right)}{\sqrt{6}}+\frac{\left(4 \cdot 2^{n}+1\right)}{\sqrt{3}}
$$

Proof. From Table 5.1 and the definition of Randic̀ index we have

$$
R\left(J_{n}\right)=\sum_{u v \in E\left(J_{n}\right)} \frac{1}{\sqrt{d(u) d(v)}}
$$

$$
\begin{aligned}
& =\frac{\left(8 \cdot 2^{n}\right)}{\sqrt{2 \cdot 1}}+\frac{\left(92 \cdot 2^{n}-5\right)}{\sqrt{2 \cdot 2}}+\frac{\left(80 \cdot 2^{n}-28\right)}{\sqrt{2 \cdot 3}}+\frac{\left(4 \cdot 2^{n}-3\right)}{\sqrt{1 \cdot 3}}+\frac{(22)}{\sqrt{3 \cdot 3}}+\frac{8}{\sqrt{3 \cdot 4}} \\
& =\frac{2^{n+3}}{\sqrt{2}}+\frac{\left(276 \cdot 2^{n}+29\right)}{6}+\frac{\left(80 \cdot 2^{n}-28\right)}{\sqrt{6}}+\frac{\left(4 \cdot 2^{n}+1\right)}{\sqrt{3}}
\end{aligned}
$$

Let $J_{n}$ be the molecular graph of the dendritic iron (II) porphyrin, then the sum-connectivity index of $I_{n}$ is given as

$$
\chi\left(J_{n}\right)=\frac{2^{n+3}}{\sqrt{3}}+\frac{\left(96 \cdot 2^{n}-8\right)}{2}+\frac{\left(80 \cdot 2^{n}-28\right)}{\sqrt{5}}+\frac{22}{\sqrt{6}}+\frac{8}{\sqrt{7}}
$$

Proof. Result is easily obtained from the definition and the Table 5.1 as

$$
\begin{aligned}
\chi\left(J_{n}\right) & =\sum_{u v \in E\left(J_{n}\right) \frac{1}{\sqrt{d(u)+d(v)}}} \\
& =\frac{\left(8 \cdot 2^{n}\right)}{\sqrt{2+1}}+\frac{\left(92 \cdot 2^{n}-5\right)}{\sqrt{2+2}}+\frac{\left(80 \cdot 2^{n}-28\right)}{\sqrt{2+3}}+\frac{\left(4 \cdot 2^{n}-3\right)}{\sqrt{1+3}}+\frac{(22)}{\sqrt{3+3}}+\frac{8}{\sqrt{3+4}} \\
& =\frac{2^{n+3}}{\sqrt{3}}+\frac{\left(96 \cdot 2^{n}-8\right)}{2}+\frac{\left(80 \cdot 2^{n}-28\right)}{\sqrt{5}}+\frac{22}{\sqrt{6}}+\frac{8}{\sqrt{7}}
\end{aligned}
$$




\section{References}

[1] S. S. Alamoti, M. Alaeiyan, and A. R. Gilani, "Studying thermodynamic properties of linear acenes molecules $\left(\mathrm{C}_{4 \mathrm{n}+2} \mathrm{H}_{2 \mathrm{n}+4}\right)$ using hyper-Zagreb index", Journal of discrete mathematical science and cryptography, vol. 22, no. 7, pp. 1261-1268, Dec. 2019, doi: $10.1080 / 09720529.2019 .1694258$

[2] A. Q. Baig, M. Naeem, W. Gao, and J.-B. Liu, "General fifth M-Zagreb indices and fifth M-Zagreb polynomials of carbon graphite", Eurasian chemical communications, vol. 2, no. 3, pp. 634-640, Feb. 2020, doi:10.33945/SAMI/ECC.2020.5.10

[3] M. Alaeiyan, C. Natarajan, G. Sathiamoorthy, and M. R. Farahani, "The eccentric connectivity index of polycyclic aromatic hydrocarbons (PAHs)", Eurasian chemical communications, vol. 2, no. 6, pp. 646-651, Mar. 2020, doi: 10.33945/SAMI/ECC.2020.6.1

[4] S. Ahmad, H.M. A. Siddiqui, A. Ali, M.R. Farahani, M. Imran, and I. N. Cangul. "On Wiener index and Wiener polarity index of some polyomino chains", Journal of discrete mathematical science and cryptograghy, vol. 22, no. 7, pp. 1151-1164, 2019, doi: 10.1080/09720529.2019.1688965

[5] A. T. Balaban, "Topological indices based on topological distances in molecular graphs", Pure and applied chemistry, vol. 55, no. 2, pp. 199206, Jan. 1983, doi: 10.1351/pac198855020199

[6] M. Cancan, S. Ediz and M. R. Farahani, "On ve-degree atom-bond connectivity, sum-connectivity, geometric-arithmetic and harmonic indices of copper oxide", Eurasian chemical communication, vol. 2, no. 5, pp. 641645, May 2020, doi: 10.33945/SAMI/ECC.2020.5.11

[7] J. Devillers and A. T. Balaban, Eds., Topological indices and related descriptors in QSAR and QSPR. Singapure: Gordon and Breach Science Publishers, 1999, doi: 10.1021/ci010441h

[8] I. Gutman and N. Trinajstić, "Graph theory and molecular orbitals. Total $\varphi$-electron energy of alternant hydrocarbons", Chemical physics letters, vol. 17, no.4, pp. 535-538, Dec. 1972, doi: 10.1016/0009-2614(72)85099-1 
[9] I. Gutman, B. Ruščić, N. Trinajstić, and C. F. Wilcox, "Graph theory and molecular orbitals. XII. Acyclic polyenes", The journal chemical physics, vol. 62, no. 2, pp. 3399-3405, 1975, doi: $10.1063 / 1.430994$

[10] S. M. Hosamani, S. G. Gadinaik, M. H. Kambar, and N. Padmannavar, "QSPR analysis of certain distance and degree-distance based topological indices", in press.

[11] H. Hosoya, "Topological index: a newly proposed quantity characterizing the topological nature of structural isomers of saturated hydrocarbons", Bulletin of the Chemical Society of Japan, vol. 44, no. 9, pp. 2332-2339, 1971, doi: 10.1246/bcsj.44.2332

[12] M. Imran, A. A. E. Abunamous, D. Adi, S. H. Rafique, A.Q. Baig, and M. R. Farahani, "Eccentricity based topological indices of honeycomb networks", Journal of discrete mathematical science and crypography, vol. 22, no. 7, pp. 1199-1213, 2019, doi: 10.1080/09720529.2019.1691326

[13] M. Imran, S. A. H. Bokhary, S.Manzoor, and M. K. Siddiqui, "On molecular topological descriptors of certain families of nanostar dendrimers", Eurasian chemical communication, vol. 2, no. 6, pp. 680-687, Jun. 2020, doi: 10.33945/SAMI/ECC.2020.6.5

[14] R. H. Jin, T. Aida, and S. Inoue, "Caged porphyrin: the first dendritic molecule having a core photochemical functionality", Journal chemical society, chemical communication, no. 16, 1993, doi: $10.1039 / \mathrm{C} 39930001260$

[15] N. Kezele, L. Klasinc, J. V. Knop, S. Ivaniš, and S. Nikolić, "Computing the variable vertex connectivity index", Croatica chemica acta, vol. 75, no. 2, pp. 651-661, 2002. [On line]. Available: https://bit.ly/3gDKSnT

[16] B. Lučić and N. Trinajstić, "New developments in QSPR/QSAR modeling based on topological indices", SAR and QSAR in environmental research, vol. 7, no. 1-4, pp. 45-62, 1997, doi: 10.1080/10629369708039124

[17] M. Kimura, Y. Nakano, N. Adachi, Y. Tatewaki, H. Shirai, and N. Kobayashi, "Intramolecular axial ligation of zinc porphyrin cores with triazole links within dendrimers", Chemistry a europen journal, vol. 15, no. 11, pp. 2617-2624, Mar. 2009, doi: 10.1002/chem.200801557 
[18] J. B. Liu, C. Wang, S. Wang, and B. Wei, "Zagreb Indices and Multiplicative Zagreb Indices of Eulerian Graphs", Bulletin Malaysian Mathematical Science Society, vol. 42, pp. 67-78, 2019, doi: 10.1007/s40840-017$0463-2$

[19] J. B. Liu, J. Zhao, H. He, and Z. Shao, "Valency-based topological descriptors and structural property of the generalized Sierpiński networks", Journal of statistical physics, vol. 177, pp. 1131-1147, Dec. 2019, doi: 10.1007/s10955-019-02412-2

[20] W. Maes and W. Dehaen, "Synthetic aspects of porphyrin dendrimers", European journal of organic chemistry, pp. 4719-4752, Oct. 2009, doi: 10.1002/ejoc.200900512

[21] G. R. Newkome, C. N. Moorefield, and F. Vögtle, Dendrimers and dendrons: concepts, syntheses, applications. Weinheim: Wiley-VCH, 2001, doi: $10.1002 / 3527600612$

[22] S. Nikolić, N. Trinajstić, and I. Baučić "Comparison between the vertex and edge connectivity indices for benzenoid hydrocarbons", Journal chemical information and computer sciences, vol. 38, no.1, pp. 42-46, Jan. 1998, doi: 10.1021/ci970031m

[23] M. Randić, "On characterization of molecular attributes", Acta chimica slovenica, vol. 45, no. 3, pp. 239-252, 1998. [On line]. Available: https://bitly/2VRZ1G0

[24] M. Randić, "Quantitative Structure property relationship: boiling points of planar benzenoids", New journal of chemistry, vol. 20, pp. 1001-1009, 1996.

[25] M. Randić and S. C. Basak, "Multiple regression analysis with optimal molecular descriptors", SAR and QSAR in environmental research, vol. 11, no. 1, pp. 1-23, 2000, doi: 10.1080/10629360008033226

[26] N. Trinajstić, S. Nikolić, D. Babic, and Z. Mihalić, "The vertex and edge connectivity indices of platonic and archimedean molecules", Bulletin of the chemists and technologists of Macedonia, vol.16, no. 1, pp.43-51,1997. [On line].Available: https://bitly/3f6u8VS

[27] B. Zhou and N. Trinajstić, "On a novel connectivity index", Journal mathematical chemistry, vol. 46, pp. 1252-1270, 2009, doi: 10.1007/s10910-008-9515-z 